\title{
Reversal of end-stage renal disease after aortic dissection using renal artery stent: a case report Andrew S Weiss ${ }^{1}$, Michael Ludkowski² ${ }^{2}$ and Chirag R Parikh*3
}

\begin{abstract}
Address: ${ }^{1}$ Department of Medicine, Internal Medicine Resident, University of Colorado Health Sciences Center, 4200 East Ninth Ave, Box B177, Denver, CO. 80262, USA, ${ }^{2}$ Department of Radiology, Assistant Professor, University of Colorado Health Sciences Center, 4200 East Ninth Ave. Box C276, Denver, CO. 80262, USA and ${ }^{3}$ Division of Renal Diseases and Hypertension, Assistant Professor, University of Colorado Health Sciences Center, 4200 East Ninth Ave. Box C281, Denver, CO. 80262, USA
\end{abstract}

Email: Andrew S Weiss - andrew.weiss@uchsc.edu; Michael Ludkowski - michael.ludkowski@uchsc.ed; Chirag R Parikh* - chirag.parikh@uchsc.edu

* Corresponding author

Published: 04 May 2004

BMC Nephrology 2004, 5:7
Received: 30 November 2003

Accepted: 04 May 2004

This article is available from: http://www.biomedcentral.com/I47/-2369/5/7

(C) 2004 Weiss et al; licensee BioMed Central Ltd. This is an Open Access article: verbatim copying and redistribution of this article are permitted in all media for any purpose, provided this notice is preserved along with the article's original URL.

\begin{abstract}
Background: Medical management is the conventional treatment for Stanford Type B aortic dissections as surgery is associated with significant morbidity and mortality. The advent of endovascular interventional techniques has revived interest in treating end-organ complications of Type B aortic dissection. We describe a patient who benefited from endovascular repair of renal artery stenosis caused by a dissection flap, which resulted in reversal of his end-stage renal disease (ESRD).

Case presentation: A 69 y/o male with a Type B aortic dissection diagnosed two months earlier was found to have a serum creatinine of $15.2 \mathrm{mg} / \mathrm{dL}(1343.7 \mu \mathrm{mol} / \mathrm{L})$ on routine visit to his primary care physician. An MRA demonstrated a rightward spiraling aortic dissection flap involving the origins of the celiac artery, superior mesenteric artery, and both renal arteries. The right renal artery arose from the false lumen with lack of blood flow to the right kidney. The left renal artery arose from the true lumen, but an intimal dissection flap appeared to be causing an intermittent stenosis of the left renal artery with compromised blood flow to the left kidney. Endovascular reconstruction with of the left renal artery with stent placement was performed. Hemodialysis was successfully discontinued six weeks after stent placement.

Conclusion: Percutaneous intervention provides a promising alternative for patients with Type $B$ aortic dissections when medical treatment will not improve the likelihood of meaningful recovery and surgery entails too great a risk. Nephrologists should therefore be aggressive in the workup of ischemic renal failure associated with aortic dissection as percutaneous intervention may reverse the effects of renal failure in this population.
\end{abstract}

\section{Background}

Medical management is the conventional treatment for most Stanford Type B aortic dissections. Surgical intervention is warranted when the dissection is thought to be rapidly expanding with impending rupture, or when there is impairment of blood flow to organs or limbs [1]. Renal ischemia is a major complication of aortic dissection occurring anywhere from $8 \%$ to $60 \%$ of presentations $[2,3]$. One study revealed that operative risk increased from $23 \%$ to $80 \%$ when renal or visceral ischemia was 
present [4]. Another study looking at operative risk for aortic dissection presenting with peripheral vascular complications revealed that impaired renal function was the only complication that was a significant independent predictor of increased operative mortality [2]. The advent of endovascular techniques has revived interest in treating the end-organ complications associated with aortic dissection [5]. The following case report describes a patient with a Type B aortic dissection who benefited from endovascular repair of a renal artery stenosis caused by a dissection flap.

\section{Case presentation}

$\mathrm{RM}$ is a 69 yo Caucasian male who was first admitted to an outside hospital in July 2002 with acute onset of severe infrascapular back pain. During this hospital admission, a CT scan with intravenous contrast revealed an aortic dissection extending to the right common iliac artery. A transesophageal echocardiogram showed a dissection flap originating at the level of the left subclavian artery and proceeding distally without any involvement of the ascending aorta or aortic valve. A Type $\mathrm{B}$ aortic dissection was confirmed. The decision was made at that time to treat the patient medically.

Soon after admission, the patient's creatinine began to rise from a baseline of $1.2 \mathrm{mg} / \mathrm{dL}(106.1 \mu \mathrm{mol} / \mathrm{L})$ and peaked at $2.3 \mathrm{mg} / \mathrm{dL}(203.3 \mu \mathrm{mol} / \mathrm{L})$ during the hospital stay. A bilateral renal ultrasound with doppler imaging revealed a right kidney measuring $10.2 \mathrm{~cm}$ with no arterial or venous flow identified. The left kidney measured $10.9 \mathrm{~cm}$ with both venous and arterial flow identified. Review of the CT scan confirmed that the patient had no perfusion of his right kidney at the time of presentation (Figures 1a and $1 \mathrm{~b}$ ). Since the patient had no history of renal dysfunction prior to this admission, he was thought to have suffered an infarction of his right kidney secondary to ischemia following aortic dissection. Further imaging by magnetic resonance angiogram (MRA) demonstrated no enhancement of the right renal artery with contrast. Without intervention, the patient's renal function recovered significantly to a level of $1.5 \mathrm{mg} / \mathrm{dl}(132.6 \mu \mathrm{mol} / \mathrm{L})$ at the time of discharge.

In September 2002, the patient was found to have a serum creatinine of $15.2 \mathrm{mg} / \mathrm{dl}(1343.7 \mu \mathrm{mol} / \mathrm{L})$ on routine follow up with his primary care physician. At the appointment, the patient complained of intermittent diffuse abdominal pain associated with eating and decreased per oral intake for ten days. The patient denied any urinary symptoms. He was subsequently admitted to our hospital for further evaluation of acute renal failure.

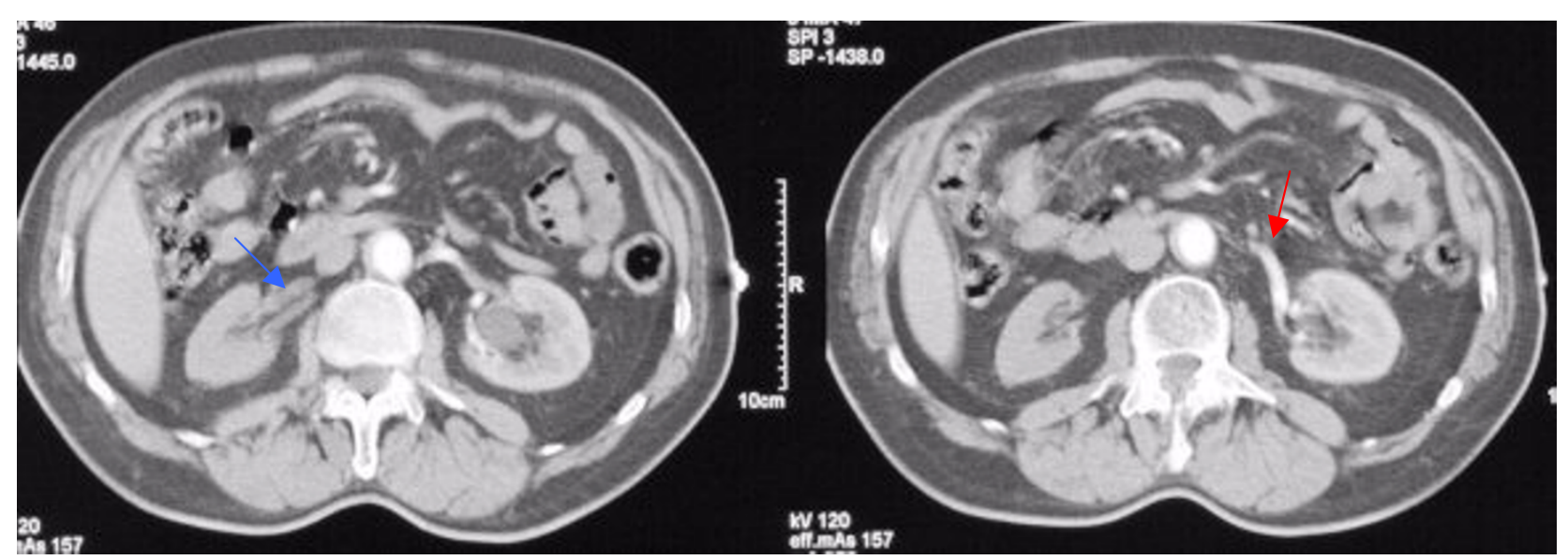

\section{Figures I}

July 2002 CT scan with intravenous contrast: Figure la shows no contrast in the right renal artery (blue arrow). Figure Ib nicely highlights contrast uptake by the left renal artery (red arrow). Both figures demonstrate atrophy of the right kidney while the left kidney enhances with contrast. 
The patient's medical history consisted of hypertension for fourteen years, dyspepsia, erectile dysfunction, and appendectomy. Medications upon admission included losartan $100 \mathrm{mg}$ PO QD, isosorbide dinitrate $20 \mathrm{mg}$ PO TID, furosemide $20 \mathrm{mg}$ PO BID, omeprazole $40 \mathrm{mg}$ PO QD, metoprolol 150 md PO BID, and acetaminophen as needed. He denied tobacco use for 35 years, drank alcohol occasionally, and denied any illicit drug use. His family history was significant for two brothers with abdominal aortic aneurysms.

On physical exam, the patient was a thin pleasant, Caucasian male in no acute distress. He was afebrile, blood pressure $140 / 100$, heart rate 70 beats per minute, respirations 20 times per minute with oxygen saturation of $94 \%$ on room air. Heart and lung exams were normal. Abdominal exam revealed positive bowel sounds, mild tenderness to palpation over the mid abdomen, no rebound, or guarding. No abdominal bruits were auscultated. He had 1+ radial, femoral and pedal pulse bilaterally with strength and sensation intact.

Significant laboratory data upon admission revealed a blood urea nitrogen of $98 \mathrm{mg} / \mathrm{dL}(35 \mu \mathrm{mol} / \mathrm{L})$, a serum creatinine of $16.4 \mathrm{mg} / \mathrm{dL}(1449.8 \mu \mathrm{mol} / \mathrm{L})$, a serum bicarbonate of $18 \mathrm{mmol} / \mathrm{L}$, and a serum potassium of 4.7 $\mathrm{mmol} / \mathrm{L}$. Troponin I was normal and electrocardiogram showed normal sinus rhythm with $1^{\text {st }}$ degree AV block. Serum hematocrit was $27.2 \%$ and peripheral smear showed no schistocytes. Urinalysis showed a specific gravity of $1.025, \mathrm{PH}$ of $5.5,30 \mathrm{mg} / \mathrm{dl}$ of protein, trace ketones, small LE. Urine sediment showed 1-2 granular casts per high powered field, 1-2 reticuloendothelial cells per high powered filed with too numerous to count red blood cells and white blood cells per high powered field.

\section{Hospital course}

Renal consultation was obtained upon admission. The differential diagnosis for the patient's acute renal failure included prerenal azotemia secondary to losartan and diuretic use, acute interstitial nephritis secondary to medications, thromboembolic disease with possible cholesterol emboli causing renal ischemia, or ischemia involving both kidneys secondary to the dissection. The patient's losartan and furosemide were discontinued and metoprolol and isosorbide dinitrate were titrated for blood pressure control. Hansel's staining of the urine showed no evidence of eosinophils and serum complement levels were normal making acute interstitial nephritis or cholesterol emboli unlikely. Renal ultrasound showed no evidence of hydronephrosis but his right kidney had decreased in size from $10.2 \mathrm{~cm}$ in July to $8.8 \mathrm{~cm}$ in length with substantial evidence of cortical thinning. The left kidney was also mildly decreased in size from $10.9 \mathrm{~cm}$ in July to $10.0 \mathrm{~cm}$ on this admission. A dynamic

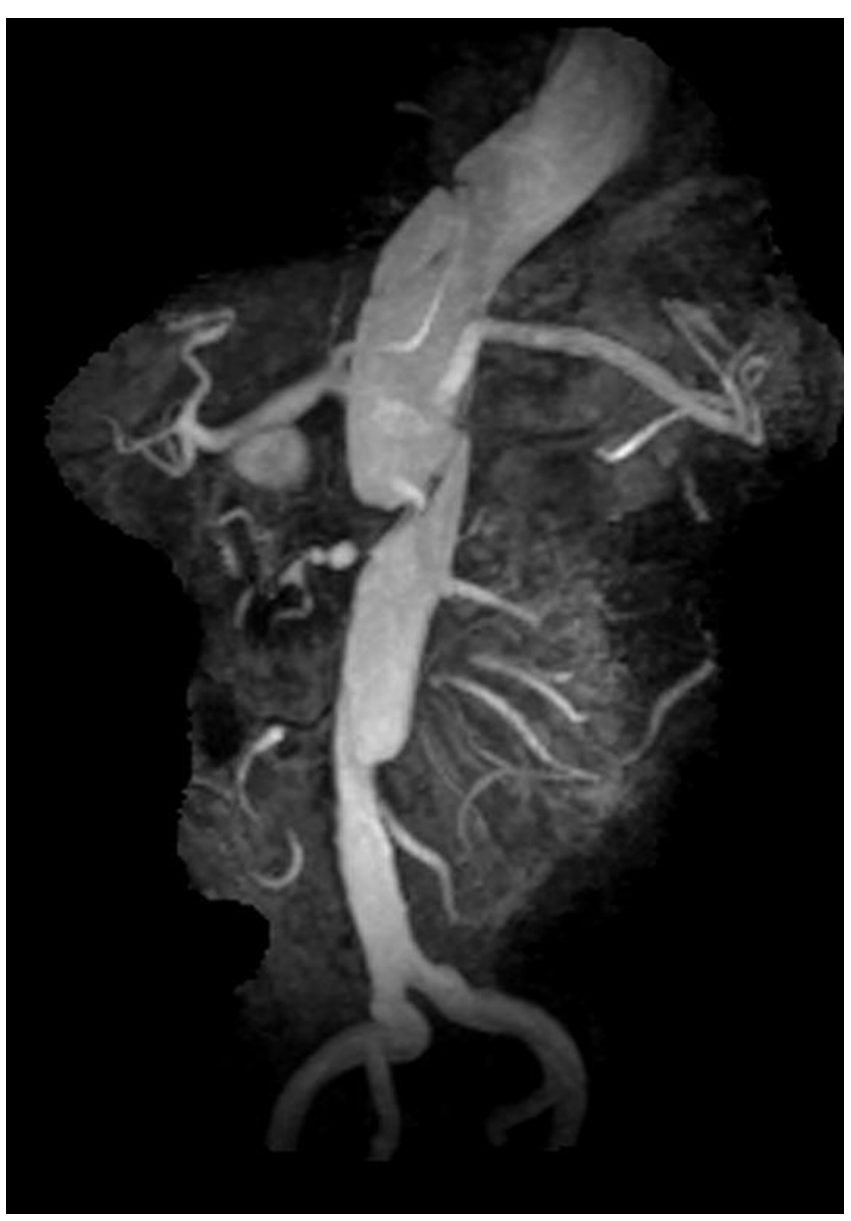

Figure 2

September 2002 magnetic resonance angiography demonstrates rightward spiraling dissection flap that involves the origins of the celiac, superior mesenteric and right renal arteries.

enhanced MRA of the abdomen revealed an aneurysmal dilatation of the suprarenal aorta with the abdominal aorta containing a rightward spiraling dissection flap, involving the origins of the celiac axis, SMA and right renal artery (Figure 2). The right renal artery was thought to be arising from the false lumen of the aorta. The left renal artery arose from the true lumen of the aorta, but an intimal flap appeared to be compressing the origin of the left renal artery.

In summary, it was felt that the patient had likely infarcted his right kidney previously as evidenced by the current renal ultrasound showing no Doppler flow and overall decrease in size of the right kidney since July. The right renal artery was now being supplied by blood from the false lumen. The right kidney exhibited severe atrophy 


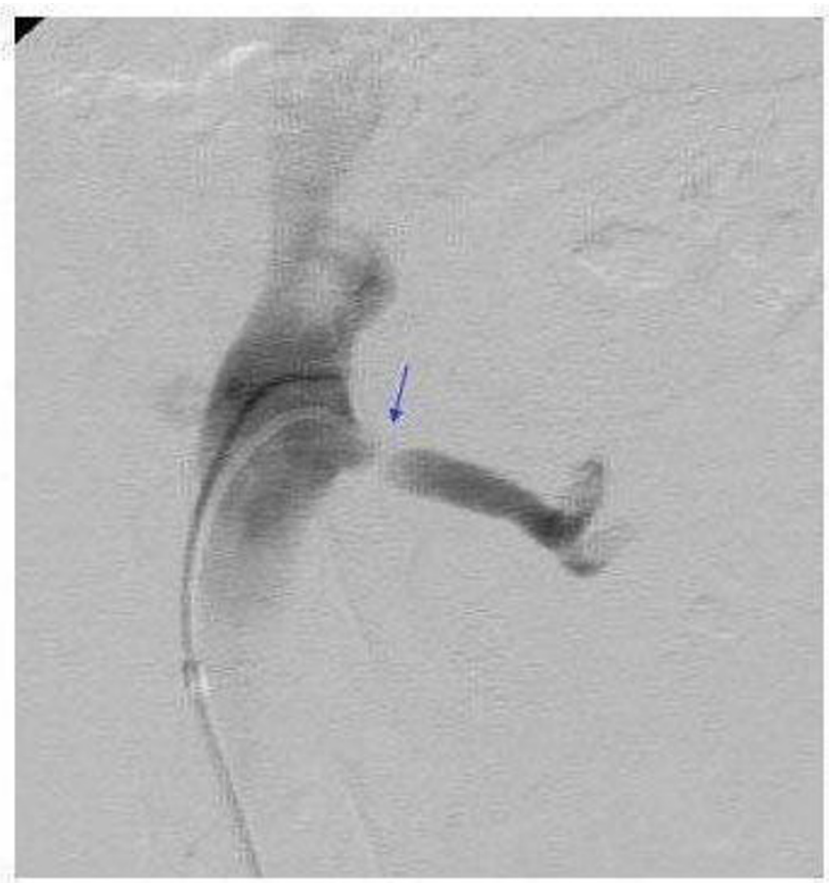

\section{Figure 3}

Left renal arteriogram reveals stenosis of the left renal artery prior to stent placement (blue arrow).

and cortical thinning and was non-functioning. The left renal artery arose from the true lumen. The recent worsening of renal function was felt to be due to additional compromise of the left renal artery by extension of the aortic dissection flap. Endovascular reconstruction of the compressed left renal artery was performed with stent placement with the hope of restoring adequate flow to the left kidney.

\section{Interventional procedure}

Arterial access to the right common femoral artery was made in the usual fashion. Diagnostic aortography was then performed. A Magic Torque measuring guidewire (Boston Scientific, Natick, MA) was placed into the left renal artery and a left renal arteriogram was performed through a 7 french Balkin sheath (Cook Inc., Bloomington, IA) (Figure 3). Vessel diameter measurements were subsequently made (Phillips Medical Systems, Integris V3000, Andover, MA). The sheath was used to traverse the stenosis and facilitate delivery of a Palmaz Genesis 154 stent (Cordis Endovascular, Warren, NJ) which was deployed on a $7 \mathrm{~mm}$ by $20 \mathrm{~mm}$ OptaPro balloon catheter (Cordis Endovascular, Warren, NJ). Post-stent deployment angioplasty was not performed. Post-stent arteriography demonstrated resolution of the left renal artery

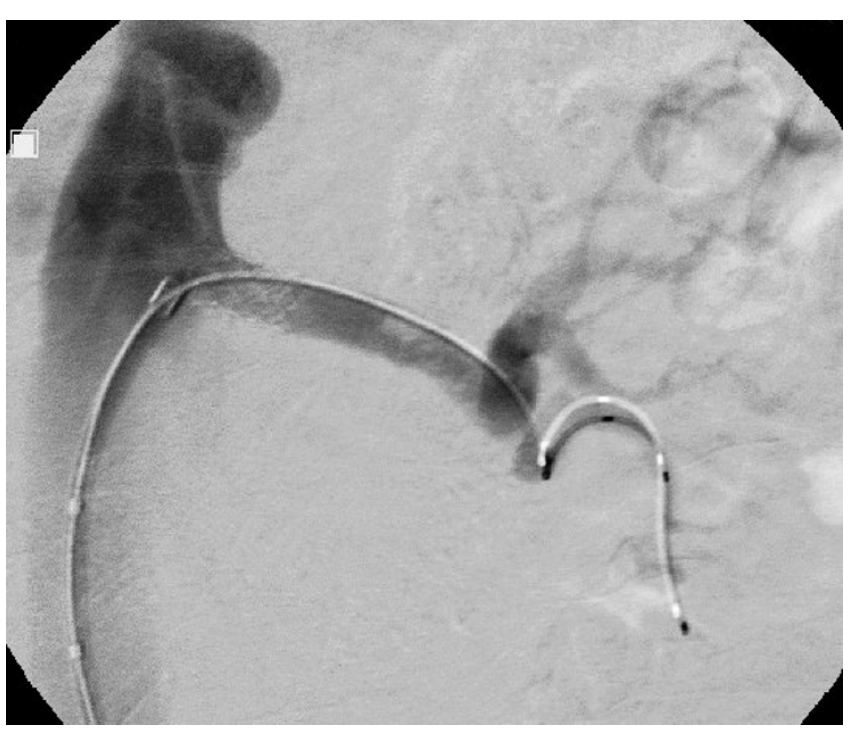

Figure 4

Post-stent arteriography demonstrates restoration of blood flow to the left kidney.

ostial stenosis. The stent extended $1 \mathrm{~mm}$ into the true lumen of the aorta and approximately $1.5 \mathrm{~cm}$ into the normal portion of the left renal artery. After the stent placement, angiography revealed improved blood flow to the left kidney (Figure 4).

Four days prior to placement of the left renal artery stent, the patient was started on hemodialysis which he continued for the next six weeks. The patient was discharged from the hospital in the middle of September with a serum creatinine level of $6 \mathrm{mg} / \mathrm{dL}(530.4 \mu \mathrm{mol} / \mathrm{L})$. By November, the patient informed us of improved urine output and an interdialytic urine collection performed at that time showed a creatinine clearance of $22 \mathrm{~mL} / \mathrm{min}$. The decision was subsequently made to discontinue hemodialysis. The patient continued to recover renal function, and now eighteen months after discontinuing hemodialysis, the patient's creatinine is $2.0 \mathrm{mg} / \mathrm{dL}(176.8$ $\mu \mathrm{mol} / \mathrm{L})$.

\section{Discussion}

The case is an example of a Type $\mathrm{B}$ acute aortic dissection with involvement of both renal arteries resulting in acute renal failure and then chronic hemodialysis. Remarkably, after endovascular reconstruction with percutaneous stent placement to the left renal artery, renal function improved significantly. After three months, the patient no longer needed hemodialysis. 
Renal ischemia is a major complication of aortic dissection occurring anywhere from $8 \%$ to $60 \%$ of presentations [2,3]. Ischemia related to aortic dissection can arise from extrinsic compression of the true lumen by the false channel or by an intimal flap compressing the orifice of the renal artery [5]. In this particular case, the right kidney suffered irreversible failure from ischemia caused by the dissection back in July. Due to this ischemia, the cortex of the right kidney atrophied as evidenced by a $2 \mathrm{~cm}$ thinning between July and September. Upon presentation in July, the patient was considered high risk for any surgical intervention. As the left kidney appeared to be uninvolved and the serum creatinine returned to near baseline prior to discharge, no interventions were considered at that time. Unfortunately, left renal ischemia progressed secondary to mechanical compression of the true lumen by the dissection flap. Restoration of blood flow to the left kidney via the true lumen by stent placement resulted in recovery of enough renal function to obviate the need for continued hemodialysis.

Four different techniques have been implemented to restore blood flow after aortic dissection. One employs endovascular stent placement within the involved renal artery to restore flow to the true lumen. The second involves endovascular fenestration of the dissection flap/ septum separating the true and false lumen. This fenestration decreases the pressure gradient between true and false lumens, thereby minimizing the "billowing effect"of the septum. A third technique uses a bare aortic stent within the true lumen of the aorta to prop open the true lumen and inhibit it from collapsing under the pressure of the expanding false lumen. A fourth technique entails covering the tear at its origin within the aorta using a covered stent graft to redirect flow into the true lumen [6].

A case report by Kammerl et al. demonstrates the use of two of these techniques in the reversal of apparent end stage renal disease from aortic dissection by successful percutaneous intervention. The case documented a patient with type B aortic dissection involving the left renal artery and known nephrosclerosis of the right kidney. Blood flow to the left kidney was severely compromised by flow through a false lumen. Fenestration of the dissection flap followed by stent placement to the left renal artery restored blood flow to the left kidney. The patient had been on hemodialysis for 2.5 months prior to the intervention and subsequently recovered enough renal function to discontinue hemodialysis shortly after the intervention [7]. Three other cases reports have documented utilization of percutaneous intervention to the renal arteries to halt ischemia- related renal injury caused by aortic dissection, but none of these patients had suffered from apparent end-stage renal disease prior to the intervention $[5,8,9]$. Our case documents a patient with ischemic involvement of both kidneys as a consequence of dissection with reversal of apparent end-stage renal disease after endovascular reconstruction with stent placement to the renal artery.

In retrospect, percutaneous intervention may have also saved the right kidney upon presentation back in July. However, medical management led to the patient's stabilization and recovery from acute renal failure at that time. It is our view that percutaneous intervention should be pursued as a treatment option at the outset when irreversible ischemic renal injury follows diagnosis of an aortic dissection.

\section{Conclusion}

Medical treatment continues to be conventional therapy for patients with type B aortic dissections. Such dissections are often complicated by ischemic renal disease, and not uncommonly, renal replacement therapy. Quality of life is significantly compromised for those patients that need renal replacement therapy as the associated morbidity is high. With these facts in mind, percutaneous intervention provides us with a promising alternative in patients when medical treatment will not improve the likelihood of meaningful renal recovery and surgery entails too great a risk. Nephrologists should therefore be aggressive in the workup of ischemic renal failure associated with aortic dissection as percutaneous intervention may reverse the effects of renal failure in this population.

\section{Competing interests}

None declared.

\section{Acknowledgements}

Written consent was obtained from the patient for this case report.

\section{References}

I. Khan I, Nair CK: Clinical, diagnostic, and management perspectives of aortic dissection. Chest 2002, 122:21 I-328.

2. Fann II, Sarris GE, Mitchell RS, et al:: Treatment of patients with aortic dissection presenting with peripheral vascular complications. Ann Surg 1990, 2 I 2:705-13.

3. Siegelman SS, Sprayregen S, Strasberg Z, et al:: Aortic dissection and the left renal artery. Radiology 1970, 95:73-78.

4. Miller DC: The continuing dilemma concerning medical versus surgical management for patients with acute type B dissections. Semin Thorac Cardiovasc Surg 1993, 5:33-46.

5. Walker PJ, Dake MD, Mitchell RS, et al.: The use of endovascular techniques for treatment of complications of aortic dissection. Journal of Vascular Surgury 1993, 18:1042-105I.

6. Slonim SM, Miller DC, Mitchell RS, et al.: Percutaneous balloon fenestration and stenting for life-threatening ischemic complications in patients with acute aortic dissection. J Thorac Cardiovasc Surg 1999, II 7: I | I 8-1 I 27.

7. Kammerl MC, Manke C, Feuerback S, et al.: Cure of apparent endstage renal disease in a patient with dissecting aneurysm of the aorta using a percutaneous interventional approach. Nephrol Dial Transplant 1999, 14:1568-1570.

8. Behrendt P, Do D, Baumgartner I, et al:: Renal artery stenting following acute aortic dissection: implantation and follow up. VASA 2000, 29:138-140. 
9. Lacombe $P$, Mulot $R$, Labedan F, et al:: Percutaneous recanalization of a renal artery in aortic dissection. Radiology 1992, |85:829-83|.

\section{Pre-publication history}

The pre-publication history for this paper can be accessed here:

http://www.biomedcentral.com/1471-2369/5/7/prepub

Publish with Bio Med Central and every scientist can read your work free of charge

"BioMed Central will be the most significant development for disseminating the results of biomedical research in our lifetime. " Sir Paul Nurse, Cancer Research UK

Your research papers will be:

- available free of charge to the entire biomedical community

- peer reviewed and published immediately upon acceptance

- cited in PubMed and archived on PubMed Central

- yours - you keep the copyright 